\title{
Compiler as Service over Cloud
}

\author{
Suryawanshi Harshal \\ Pune University \\ Computer Engineering \\ PREC, Loni
}

\author{
Rokade Chakrapani \\ Pune University \\ Computer Engineering \\ PREC, Loni
}

\author{
Ambhore Ajay \\ Pune University \\ Computer Engineering \\ PREC, Loni
}

\author{
Rathod Sharad \\ Pune University \\ Computer Engineering \\ PREC, Loni
}

\begin{abstract}
In the today's world everything is online so we are creating software for the today's faster world called compiler as service over the cloud. Cloud computing refers to the logical computational resources (data, software) accessible via a computer network (through WAN or Internet etc.), rather than from a local computer. Data are stored on Server Farms generally located in the country of the service provider. The paper aims to describe an online compiler which helps to reduce the problems of portability and storage space by making use of the concept of cloud computing. The ability to use different compilers allows a programmer to pick up the fastest or the most convenient tool to compile the code and remove the errors. This project is being developed for JAVA \& .NET languages. Moreover, a web-based application can be used remotely throughout any network connection and it is platform independent. The problem of several compiler installations can be eliminated. Also error/output are stored in convenient way..
\end{abstract}

Keyword:- cloud computing, compiler

\section{INTRODUCTION}

Cloud computing implies a service oriented architecture, reduced information technology overhead for the end-user, great flexibility, reduced total cost of ownership and ondemand services among other advantages. The National Institute of Standards and Technology (NIST) defines 'Cloud Computing' as 'a model for enabling easy, on-demand network access to a shared pool of configurable computing resources (e.g., networks, servers, storage, applications, and services) that can be rapidly provisioned and released with minimal management effort or service provider interaction.' Cloud computing differs from the classic client-server model by providing applications from a server that are executed and managed by a client's web browser, with no installed client version of an application required. Centralization gives cloud service providers complete control over the versions of the browser-based applications provided to clients, which removes the need for version upgrades or license management on individual client computing devices. The phrase "software as a service" (SaaS) is sometimes used to describe application programs offered through cloud computing.

A compiler, which is the heart of any computing system, transforms source code from a higher level language to a lower, machine level language. This is mainly done in order to create executable files which can then be 'run' in order to

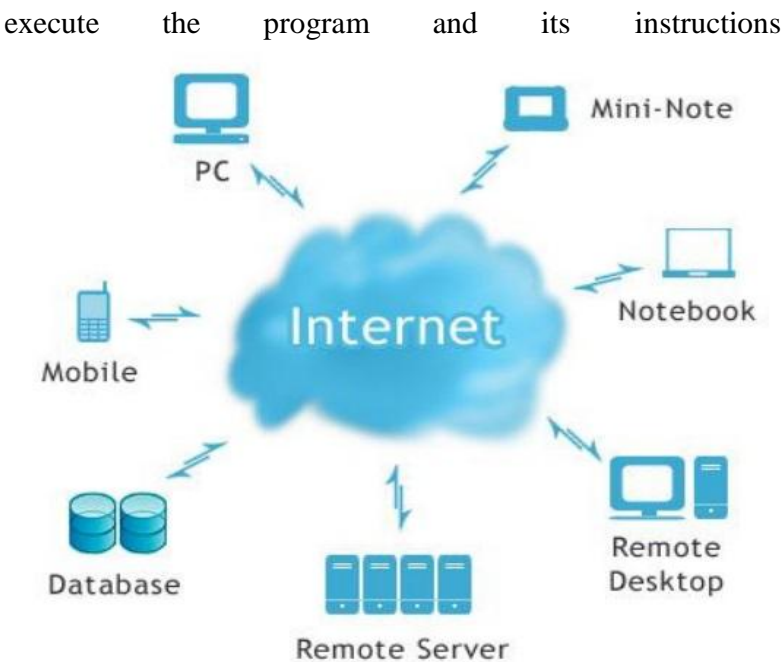

Fig No 1: Cloud Computing

\section{NEED FOR PROJECT}

The main advantage of cloud computing over the other nonnetwork methods is of faster processing. Also, many processors can be used remotely, without the knowledge of the user(s), in order to expedite the processing. Thus, keeping this main advantage in mind, the main reason for creating the project is to provide a centralized compiling scheme for organizations or institutions. Also, it will act as a centralized repository for all the codes written. The other major advantage that this system will have over the others is that it will make the users system lightweight i.e. there will be no need to maintain separate compilers/SDK's at the client-side. Thus, for educational institutions this will prove to be highly efficient. Also, the process of maintenance and distribution of dynamic usernames and passwords will be greatly simplified. Also, authentication and personalized task distribution will be made possible.

\section{COMPILER AS SERVICE OVER CLOUD}

Using compiler as service over cloud, all programs and their timestamps are stored at server side and also compiled at server side. This project is provided with one application using this application user can login and then write the code in window or directly import form another location of his local drive. User also has facility to choose language either JAVA or .NET. Then by using the compile and execute option code is compile and any error is generated then this report is displayed on client window. There is no interaction hardware directly, as there are pointers in JAVA and .NET server can execute the program easily. 


\section{TECHNOLOGY}

According to Sun, the developer of JAVA, Java Platform, Enterprise Edition or Java EE is a widely used platform for server programming in the Java programming language. The Java platform (Enterprise Edition) differs from the Java Standard Edition Platform (JavaSE) in that it adds libraries which provide functionality to deploy fault-tolerant, distributed, multi-tier Java software, based largely on modular components running on an application server.

\section{ADVANTAGES}

This new generation of technology is based on Web services- small building-block applications that can connect to each other as well as to other, larger applications over the Internet. J2EE combines unprecedented developer productivity with performance, reliability, and deployment. J2EE makes building real world Web applications dramatically easier. GlassFish server controls enable an HTML-like style of declarative programming that let a person build great pages with far less code. Displaying data, validating user input, and uploading files are all amazingly easy. Java EE includes several API specifications, such as JDBC, RMI, e-mail, JMS, web services, XML, etc., and define show to coordinate them. Java EE also features some specifications unique to Java EE for components. These include Enterprise JavaBeans, Connectors, servlets, portlets (following the Java Portlet specification), JavaServer Pages and several web service technologies. This allows developers to create enterprise applications that are portable and scalable, and that integrate with legacy technologies.

\section{SYSTEM ARCHITECURE}

The system uses a dual-layered architecture. The lower layer consists of clients, which are of lower configuration. The upper layer consists of the server. The important components of the upper layer are describe as below:

1. GlassFish Server which handles the work of scripting and compilation of code.

2. Database which stores the client information.

3. The 'cloud hard disk' is a shared resource.

4. The "cloud compiler' is also shared resource.

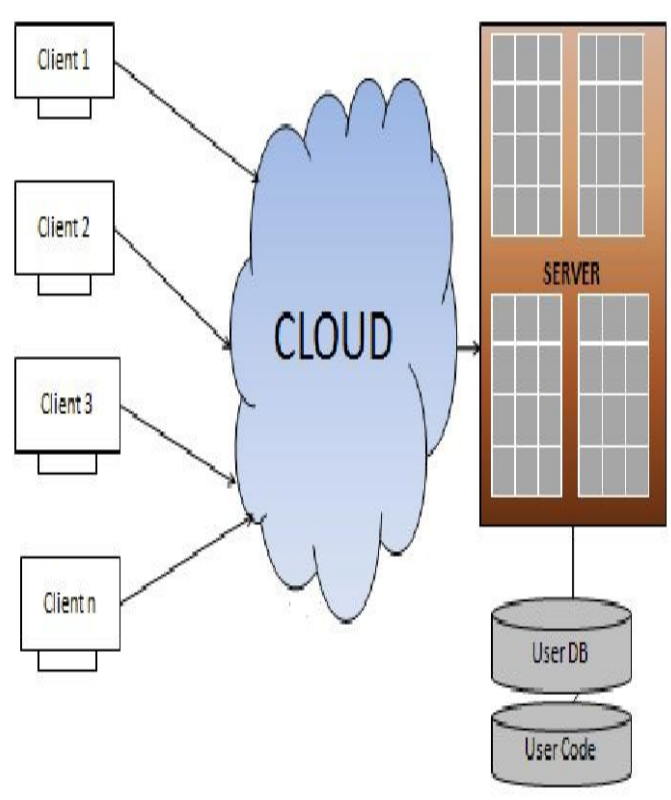

Fig No 2: Project Architecture

\section{SYSTEM DESIGN}

The primary functions of our project are:

Login Option: Using this option user can login into the cloud of the Cloud Vendor. Then user is provided with login id and password, by using these details he can login to that cloud form any network.

Compile option: This would take the code in the text box to the server side for its compilation and at the server side the compiler package has been imported.

Execute Option: The user code is being executed and result will be displayed on client window.

\section{SYSTEM IMPLEMENTION}

While developing the project main issue is to be considering that importing the particular package of compiler as need of the user. Then we develop a system in which client is login into web start application and this request is handled by a remote server. Client side programs are transfer using soap. They are executed only on the client machine and not on the server. This allows for sharing the computational cost between the server and client. 


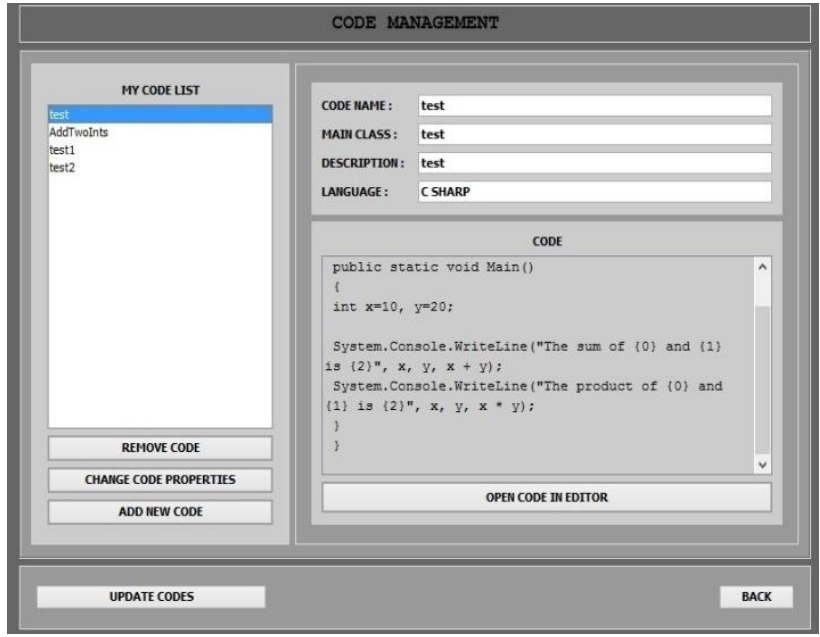

Fig 3: Adding the New code in database

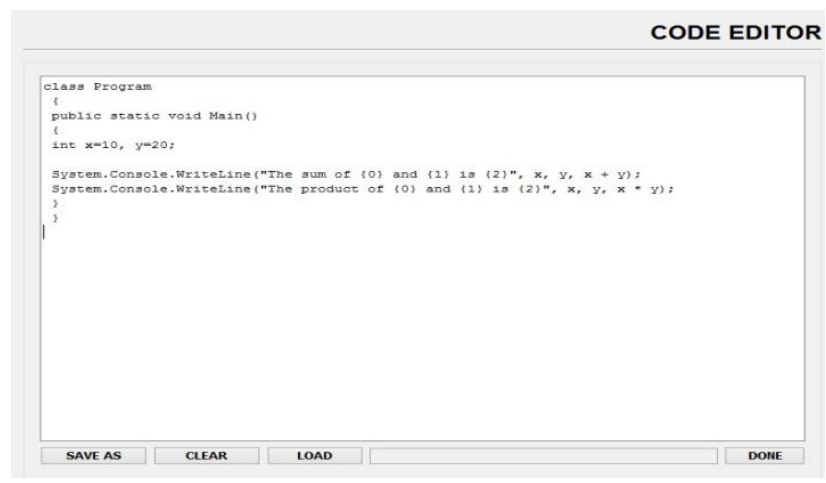

Fig 4: Editing the code

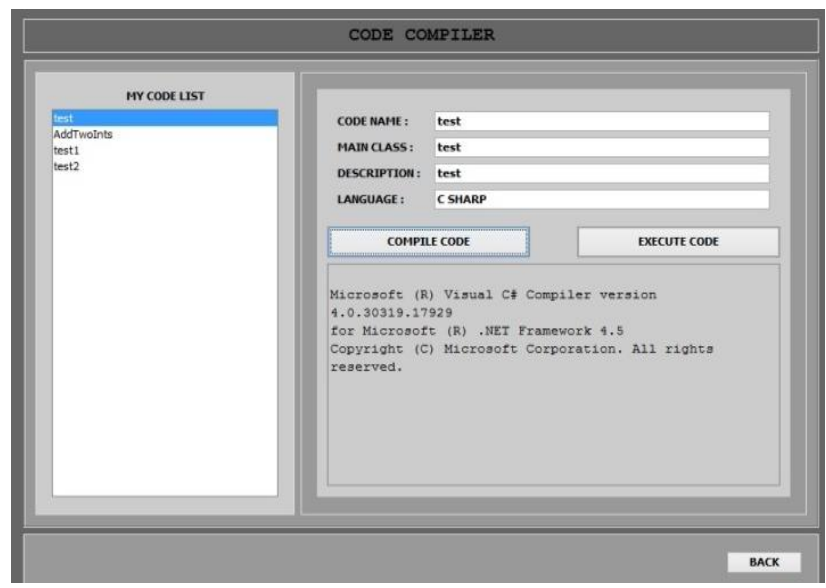

Fig 5: Compiling the code (If no any Error)

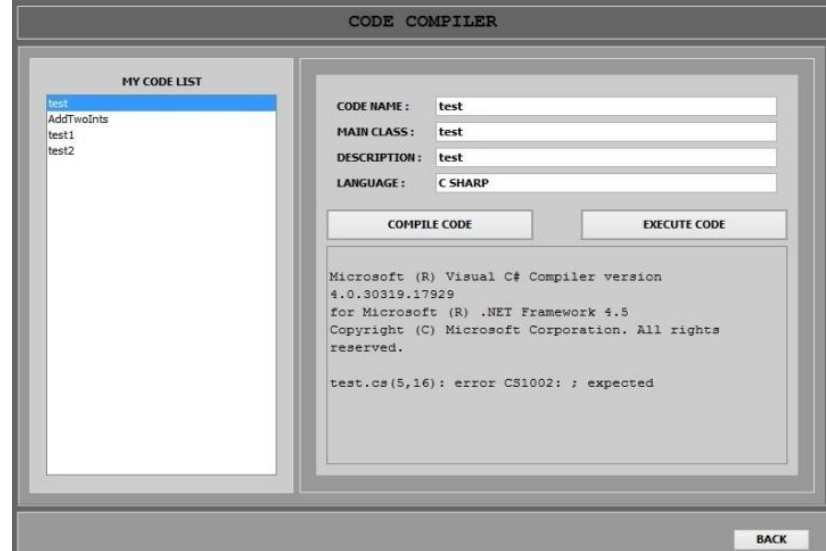

Fig 6: Error Report Generated

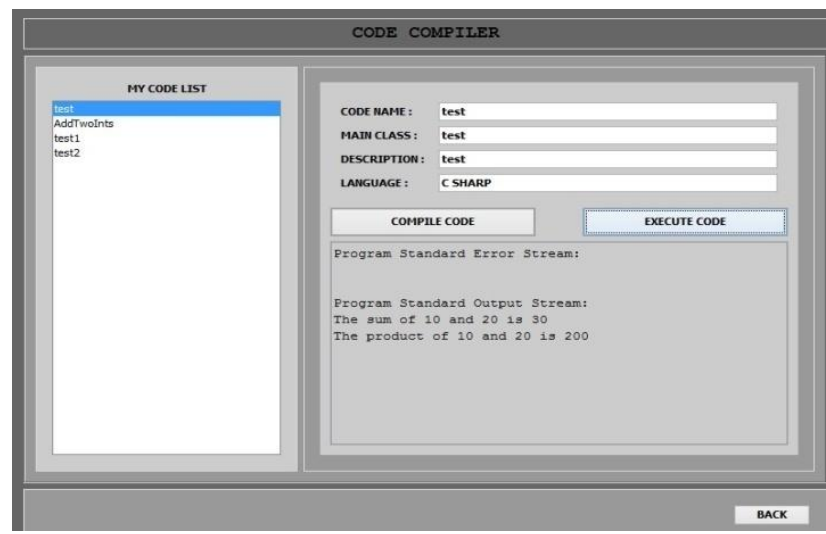

Fig 7: Executing the code

To use the COMPILER AS SERVICE OVER CLOUD user has to paste code in the provided window and as clicking on to 'Done' button the user code is sent to a remote server. User code is executed and then after execution, if any error are generated are sent to user or file will be executed.

\section{APPLICATION OF PROJECT}

The purpose of the project is provide different compiler over network. Online compiler can be used in MNC for centralized database as well as centralized compiler installation. Because of centralized compiler on the server side employee desktop should not need that much of configuration. Online compiler can be used in school/college/Institutes for practical examination also using this student can have facility to use/learn multiple compilers. Using this project one can develop multi language Cloud compiler.

\section{CONCLUSION}

By making the use of characteristics of the Cloud computing and compilers we develop a system which compile the JAVA \& .NET code.As compared to current scenario, in which if any compiler upgradation is required its done on each machine but by using above system there only centralized compilers upgraded .By leveraging Cloud technology, customers continue to use their existing compilers, while removing redundant software licenses from their environment. With no upfront investment, customers who implement Cloud 
Compiling reduce their monthly compiler fees by $50-75 \%$, while achieving greater systems controls, increased flexibility, and the ability to scale quickly with minimal notice.

\section{ACKNOWLEDGEMENT}

My most sincere thanks go to my guide, Prof. M.R.Bendre. We thank him for providing me opportunity to work in the area of online compilation for cloud. We thank him for guidance, encouragement and support during initial development of this project.

\section{REFERENCES}

[1] Online $\mathrm{C} / \mathrm{C}++$ Compiler using Cloud Computing Aamir Nizam Ansari, Siddharth Patil, Arundhati Navada, Aditya Peshave, Venkatesh Borole, Pune Institute of Computer Technology, Pune, University of Pune.

[2] Vouk, M. A., "Cloud Computing - Issues, Research and Implementations" - ITI 2008 - 30th International Conference on INFORMATION TECHNOLOGY INTERFACES.

[3] Shufen Zhang Shuai Zhang Xuebin Chen Shangzhuo, "Analysis and Research of Cloud Computing System Instance", Future Networks,2010. ICFN '10. Second International Conference. 